\title{
Iterative Transmitter and Receiver Optimization for Synchronous CDMA Systems
}

\author{
Sennur Ulukus \\ University of Maryland \\ e-mail: ulukus@eng. und.edu
}

\author{
Aylin Yener \\ Pennsylvania State University \\ e-mail: yener@ee.psu.edu
}

The user capacity and the information theoretic sum capacity of a single-cell CDMA system have been recently shown to be achieved by the same transmit strategies. We propose an alternating minimization based algorithm that updates the transmitters and the receivers of the users iteratively. The algorithm is suitable for online implementation and alleviates the need for scheduling of the user updates, both of which are in contrast with the previously proposed algorithms.

For a single-cell $K$-user synchronous CDMA system with processing gain $N$, the chip-matched filtered received signal is

$$
\mathbf{r}=\sum_{i=1}^{K} \sqrt{p_{i}} b_{i} \mathbf{s}_{i}+\mathbf{n}=\mathbf{S} \mathbf{P}^{1 / 2} \mathbf{b}+\mathbf{n}
$$

where $\mathbf{S}=\left[\mathbf{s}_{1}, \ldots, \mathbf{s}_{K}\right]$ contains the signature sequences in its columns, $\mathbf{P}=\operatorname{diag}\left\{p_{1}, \cdots, p_{K}\right\}$ is the diagonal matrix with received powers, $\mathbf{b}=\left[b_{1}, \cdots, b_{K}\right]^{\top}$ is the vector of bits, and $\mathbf{n}$ is a zero-mean Gaussian random vector with $E\left[\mathbf{n} \mathbf{n}^{\top}\right]=\sigma^{2} \mathbf{I}_{N}$.

The information theoretic sum capacity of this system is [1]

$$
C_{\text {sum }}=\frac{1}{2} \log \left[\operatorname{det}\left(\mathbf{I}_{N}+\sigma^{-2} \mathbf{S P S}^{\top}\right)\right]
$$

For equal received powers, the sum capacity is maximized by orthonormal signature sequences when $K \leq N$, and by Welch Bound Equality (WBE) sequences when $\bar{K}>N[2]$. For unequal powers, the sum capacity is maximized when the eigenvalues of SPS ${ }^{\top}$ are a Schur-minimal element [3] of the set of all feasible eigenvalues [4]. The optimum signature sequences minimize the total (weighted, for unequal powers) squared correlation, $\mathrm{T}(\mathrm{W}) \mathrm{SC}$. An iterative and distributed algorithm to construct optimum signature sequences was proposed in [5]; the algorithm is based on the principle of updating the signature sequence of one user at a time to decrease the TSC.

Total mean squared error (MSE) of the system is

$$
\mathrm{MSE}=\operatorname{tr}\left[\mathbf{C}^{\top}\left(\mathbf{S P S}^{\top}+\sigma^{2} \mathbf{I}_{N}\right) \mathbf{C}-2 \mathbf{C}^{\top} \mathbf{S} \mathbf{P}^{1 / 2}+\mathbf{I}_{K}\right]
$$

where $\mathbf{C}=\left[\mathbf{c}_{1}, \cdots, \mathbf{c}_{K}\right]$ contains the receiver filters of the users in its columns. The minimum MSE (MMSE) is

$$
\operatorname{MMSE}=K-\operatorname{tr}\left[\mathbf{S P S}^{\top}\left(\mathbf{S P S}^{\top}+\sigma^{2} \mathbf{I}_{N}\right)^{-1}\right]
$$

and the TWSC of the system is

$$
\mathrm{TWSC}=\operatorname{tr}\left[\left(\mathbf{S P S}^{\top}\right)^{2}\right]
$$

Note that $C_{\text {Sum }}$ is Schur-concave, and MMSE and TWSC are Schur-convex functions of the eigenvalues of $\mathbf{S P S}^{\top}$ [3]. Therefore, $C_{\text {sum }}$ is maximized, and MMSE and TWSC are minimized by the same set of signature sequences.

$C_{\text {sum }}$, MMSE and TWSC are functions of the signature sequences only. Since our aim is to obtain an online iterative joint transmitter-receiver update algorithm, we will concentrate on the MSE criterion given in (3) which is a function of the signature sequences as well as the receiver filters. The minimization of the MSE subject to $\mathbf{s}_{i}^{\top} \mathbf{s}_{i}=1$, for all $i$, can be accomplished using alternating minimization technique [6] where all but one variable are fixed, and the remaining variable is chosen to minimize the MSE. The updates for the receiver filters and the signature sequences are found to be,

$$
\begin{aligned}
& \mathbf{c}_{i}=\sqrt{p_{i}}\left(\mathbf{S P S}^{\top}+\sigma^{2} \mathbf{I}_{N}\right)^{-1} \mathbf{s}_{i} \\
& \mathbf{s}_{i}=\sqrt{p_{i}}\left(p_{i} \mathbf{C C}^{\top}+\alpha_{i} \mathbf{I}_{N}\right)^{-1} \mathbf{c}_{i}
\end{aligned}
$$

where $\alpha_{i}$ is chosen such that $\mathbf{s}_{i}^{\top} \mathbf{s}_{i}=1$. Since the signature sequence updates depend only on the receiver filters (the receiver filter updates depend only on the signature sequences), the users can update their signature sequences (receiver filters) simultaneously, i.e., in parallel.

The convergence of the overall algorithm is established by first observing that each update decreases the total MSE, and then noting that the fixed points of the algorithm satisfy

$$
\mathbf{S P S}^{\top} \mathbf{s}_{i}=\lambda_{i} \mathbf{s}_{i} \quad i=1, \cdots, K
$$

The fixed points of the proposed algorithm are the same as those of the MMSE algorithm [5]. The set of fixed points described by (8) includes a wide spectrum of signature sequences, ranging from the very best signature sequences that the algorithm could converge to, to the absolutely worst signature sequences $\left(s_{i}=\mathrm{s}\right.$ for all $\left.i\right)$. As in [5], we have observed that the algorithm always converged to a set of optimum signature sequences when started with a randomly generated set. This claim is supported by [7] which proves that the suboptimum fixed points of the MMSE algorithm of [5] are unstable, i.e., the iterations never converge to any one of the suboptimum fixed points unless they are started with one of them.

\section{REFERENCES}

[1] S. Verdú, "Capacity region of Gaussian CDMA channels: The symbol-synchronous case," Allerton Conf., October 1986.

[2] M. Rupf and J. L. Massey, "Optimum sequence multisets for synchronous code-division multiple-access channels," IEEE Trans. Info. Theory, 40(4):1261-1266, July 1994.

[3] A. W. Marshall and I. Olkin, Inequalities: Theory of Majorization and Its Applications, New York: Academic, 1979.

[4] P. Viswanath and V. Anantharam, "Optimal sequences and sum capacity of synchronous CDMA systems," IEEE Trans. on Info. Theory, 45(6):1984-1991, September 1999.

[5] S. Ulukus and R. D. Yates, "Iterative construction of optimum signature sequence sets in synchronous CDMA systems," IEEE Trans. on Info. Theory, 47(5):1989-1998, July 2001.

[6] D. Bertsekas, Nonlinear Programming, Athena Scientific, 1995.

[7] P. Anigstein and V. Anantharam, "Ensuring convergence of the MMSE iteration for interference avoidance to the global optimum," Allerton Conf., September 2000. 